\title{
EL TREINTA ANIVERSARIO DEL 23-F EN LA WEB 2.0: LA TEORÍA DE LA ESPIRAL DEL SILENCIO EN LA “AUDIENCIA CREATIVA"
}

Virginia Martín-Jiménez: Universidad de Valladolid. España virgimj@hmca.uva.es

Eva Campos-Domínguez: Universidad de Valladolid. España eva.campos@hmca.uva.es

\section{RESUMEN}

En un contexto marcado por el surgimiento de la denominada por Manuel Castells (2010) "audiencia creativa" y "autocomunicación de masa" y tomando en consideración los estudios de audiencias, esta investigación pretende conocer -tal y como han apuntado ya algunos autores como Neuwirth (2007), Hai-long (2001), entre otros- si la teoría de la espiral del silencio puede resultar válida también en los estudios de comunicación en Internet. Para ello, tras la pertinente revisión bibliográfica, esta investigación aborda un estudio concreto basado en el análisis de contenido descriptivo de los comentarios realizados a la noticia más leída y comentada por los usuarios sobre el fallido golpe de estado del 23-F, a raíz de su treinta aniversario, en el portal español de ranking editorial www.meneame.net, así como también los comentarios realizados sobre la propia información original una entrevista publicada en el diario Público- para tratar de verificar o refutar si, en los estudios de comportamiento de las audiencias en el ciberespacio, puede considerarse como aplicable, o no, la teoría clásica de la alemana Elisabeth NoelleNeumann.

PALABRAS CLAVE: Espiral del silencio - 23-F - Comunicación política - Internet Web 2.0

\footnotetext{
1 Autor correspondiente

Virginia Martín-Jiménez: Profesora de Periodismo. Universidad de Valladolid. Valladolid, España
} 


\title{
THE 23-F THIRTIETH ANNIVERSARY ON THE WEB 2.O: THE THEORY OF THE SPIRAL OF SILENCE IN THE “CREATIVE AUDIENCE"
}

\begin{abstract}
In a context marked by the emergence of the "creative audience" or "mass selfcommunication" (Manuel Castells, 2010) and take into consideration the audience studies, this research seeks to find -as some authors have already say: Neuwirth (2007) or Hai-long (2001-, if the theory of spiral of silence may be valid also in studies of online communication. To reach this objectives, after appropriate review of the most important studies, this research want to draw a specific study based on a content analysis of comments that users made to the news most read and commented about the 30th anniversary of the 23-F in the Spanish editorial raking web www.meneame.net, as well as comments made on the originally information -an interview published in the daily Public-to try to verify or disprove whether, in audience's behaviour studies in cyberspace can be considered as applicable, or not, the classical theory of Elisabeth Noelle-Neumann.
\end{abstract}

KEY WORDS: Spiral of silence - 23-F - Political communication - Internet - Web 2.0

\section{INTRODUCCIÓN}

La evolución que ha experimentado Internet como medio de comunicación ha promovido el surgimiento de un nuevo paradigma comunicativo. El modelo tradicional basado en un sistema jerárquico y horizontal ha sido superado gracias al mayor número de opciones de interactividad que nos proporciona la Red.

Con lo cual, frente a ese modelo unidireccional, surge la "autocomunicación de masas" de la "audiencia creativa", si empleamos el término acuñado por Manuel Castells (2010). Este tipo de comunicación destaca por su carácter más dinámico y multidireccional que posibilita que los públicos, hasta el momento pasivos, se conviertan en usuarios activos, fragmentarios y diversificados (López, 2011).

Esta investigación parte de este contexto teórico para, tomando en consideración los estudios de audiencias, reflexionar acerca de si la teoría de la espiral del silencio puede ser aplicada, obteniendo unos resultados válidos, a los estudios sobre 


\section{METODOLOGÍA}

Con este fin, y partiendo de una revisión bibliográfica previa, el presente artículo aborda un estudio concreto basado en el análisis de contenido descriptivo de los comentarios realizados a la noticia más popular y comentada por los usuarios sobre el fallido golpe de estado del 23-F, a raíz de la conmemoración de su treinta aniversario, en el portal español de ranking editorial Menéame.net, así como también los comentarios realizados sobre la propia información original -una entrevista publicada en el diario Público- para tratar de verificar o refutar si, en los estudios de comportamiento de las audiencias en el ciberespacio, puede considerarse como aplicable, o no, la teoría clásica de la alemana Elisabeth Noelle-Neumann.

\section{ANÁLISIS Y DISCUSIÓN}

\subsection{La teoría de la espiral del silencio y la web 2.0}

Para Noelle-Neumann los miembros de la sociedad tienen "el don de percibir con gran sutileza el desarrollo de las opiniones de su ambiente" (Noelle-Neumann, 1978, p. 76). A partir de esa percepción se pondrá en marcha el mecanismo psicosocial denominado por la autora, "espiral del silencio". Mecanismo que provocará que el individuo como recuerda Cándido Monzón,

la teoría de la espiral, en algunos aspectos, ya había sido tratada anteriormente bajo expresiones como ignorancia pluralista, mayoría silenciosa o efectos del vagón de cola, pero será la autora alemana, en diferentes artículos (...) y más adelante en su libro La espiral del silencio (1995), quien exponga desde el punto de vista histórico, crítico y doctrinal una nueva visión de los efectos de los medios y una nueva explicación de la formación de la opinión pública. Por un lado, estudiará la formación de la opinión pública dentro del proceso de la comunicación política, por otro, extenderá el concepto de opinión pública a fenómenos distintos de los políticos y, finalmente, recuperará los hallazgos de la psicología social opte por subirse al carro del vencedor y al hacerlo se muestre más seguro de sus ideas al sentir que están en consonancia con las que priman en su entorno $\mathrm{o}$, al contrario, al percibir que forma parte de una minoría acabe ocultándose en el silencio. Por lo tanto, para la pensadora alemana la opinión pública queda reducida a aquello que ante una controversia las personas pueden expresar públicamente sin temor a caer en el ostracismo (Noelle-Neumann, 1979, p. 151). 
Los medios de comunicación, según esta autora, son capaces de generar un clima de opinión a partir del cual se logre hacer creer a la gente que la imagen que difunden los medios es un reflejo de la realidad; lo cual queda reforzado cuando se observa el fenómeno de "consonancia irreal" al que tienden los periodistas en las sociedades democráticas y a partir del cual la información, al margen de su origen, acaba convergiendo en un único mensaje casi estereotipado.

Noelle-Neumamm, a través de encuestas y análisis de contenido mediático, llevó a cabo sus investigaciones en torno a temas como la tendencia de voto, la educación infantil o la pena de muerte. ${ }^{2}$

Los resultados de sus trabajos le condujeron a afirmar, partiendo de una explicación psicológica, que los miembros de la sociedad tienden a percibir cuál es la corriente de opinión dominante, y al entender -a través de una falsa impresión- el clima de opinión que generan los medios como la opinión mayoritaria o, en otras palabras, como la opinión pública, tratarán de reducir todo lo posible las disonancias con el entorno.

De esta manera, al quedar inhibidas ciertas al estudio de la opinión pública, cuando, por ejemplo, traslade al ámbito de lo público el modelo de presión, ajuste, o conformidad que ejercen unas personas sobre otras cuando forman parte de un grupo o una colectividad". (Monzón, 2006)

Que recibía apoyo explícito parecer[á] más fuerte de lo que era realmente, y la otra opinión más débil. Las observaciones realizadas en unos contextos se extendieron a otros e incitaron a la gente a proclamar sus opiniones o tragárselas y mantenerse en silencio hasta que, en un proceso en espiral, un punto de vista llegó a dominar la escena pública y el otro desapareció de la conciencia pública al enmudecer sus partidarios. Éste es el proceso que podemos calificar como de espiral del silencio" (Noelle-Neumann, 2003. p. 22).

\footnotetext{
2 Noelle-Neumann construyó su teoría a partir del estudio de las campañas electorales alemanas que tuvieron lugar en 1965 y en 1972. En estos dos comicios la autora percibió una marcada diferencia entre
} 
Los medios de comunicación, además de generar un determinado clima de opinión, van a cumplir una función articuladora, en el sentido de que son ellos quienes van a administrar al público los argumentos que este empleará para defender su punto de vista. De tal manera que si esos medios no le facilitan habitualmente expresiones en favor de su punto de vista, terminará enmudeciendo (Noelle-Neumann, 2003. p. 227).

Dentro del actual panorama mediático dominado por Internet y la interactividad que permite la Web 2.0, la teoría de la espiral del silencio nos ayuda a comprender qué mecanismos actúan en los procesos de autocomunicación que los usuarios llevan a cabo en plataformas participativas on-line. Tal y como señala León:

Los foros en los medios digitales están ofreciendo una imagen nueva de las audiencias; si bien desde los años 80 han venido desarrollándose los contramo de los de las audiencias activas frente a las dominantes en las décadas anteriores, sólo han tomado cuerpo esa 'actividad' de la audiencia, no controlada de intento por el medio digital que sabe que tienen en la interactividad una de sus claves de difusión (León, 2005. p. 166).

Partiendo de los planteamientos de Noelle-Neumann, cabe preguntarse si el usuario de la Web 2.0 también estará percibiendo el clima de opinión que le transmiten los medios y, por una falsa creencia, asimilará esa opinión como la mayoritaria y, por lo tanto, como equivalente a la opinión pública, de tal modo que se esté produciendo una promoción de la selectividad y la autocensura o bien de la interactividad o la expresividad (Rojas y Hopke, 2010).

De esta forma, y siguiendo la idea de la espiral del silencio, el usuario a la hora de verter sus opiniones en la Red tenderá a difundir aquellas que se asemejen a lo que él considera la opinión mayoritaria (vanguardia) o a callar por miedo a no ser aceptado por el resto de los usuarios.

Este mecanismo explicaría que los comentarios que se cuelgan en Internet presenten una línea de pensamiento muy semejante, normalmente acorde con el planteamiento editorial de cada medio o con la que se considera opinión mayoritaria, y que en el momento en el que un usuario, como actor desviado, rompa esa consonancia comenzarán a publicarse comentarios que le respalden, provenientes de aquellos que por miedo al ostracismo habían optado por el silencio o por sumarse al "carro vencedor" (opinión percibida como mayoritaria) o de quienes modificaron sus posiciones al sentir la llegada de una nueva vanguardia. 
El surgimiento de ese actor desviado es equivalente a lo que la pensadora alemana denomina "núcleo duro"; es decir:

La minoría que queda al final de un proceso de espiral del silencio desafiando la amenaza de aislamiento. El núcleo duro está, en cierto sentido, relacionado con la vanguardia, ya que considera el aislamiento como un precio que debe pagar. A diferencia de los miembros de la vanguardia, un núcleo duro puede dar la espalda al público, puede encerrarse completamente cuando se encuentra en público con desconocidos, se puede encapsular como una secta y orientarse hacia el pasado o hacia el futuro más lejano. La otra posibilidad es que el núcleo duro crea simultáneamente una vanguardia. Esto lo demuestran en su disposición a expresarse, una disposición tan intensa al menos como la de la vanguardia. (...) Y por esta razón, a pesar de representar una opinión minoritaria, est[án] dispuestos a participar en una conversación directa (NoelleNeumann, 2003. Pág. 227).

Con lo cual, si nos basamos en este concepto de opinión pública como control social, no todas las opiniones son debatidas por igual, puesto que el que exista una discusión al respecto dependerá del contexto en que dicha discusión tenga lugar. A pesar de las variables individuales que pueden determinar la puesta en marcha de otros mecanismos psicológicos, lo cierto es que la espiral del silencio puede terminar generando un proceso sociológico de autocensura que termina por encorsetar los flujos comunicacionales.

\subsection{El 23-F en los medios de 2011}

El 30 aniversario del 23-F, que tuvo lugar en 2011, se presenta como un acontecimiento conmemorativo apropiado para comparar el mensaje ciudadano en Internet con el mensaje mediático. Esta investigación parte, en su contextualización, de la referencia a la información sobre el referido evento publicada en los medios tradicionales y en sus plataformas digitales el día que se conmemoraba el fallido golpe $^{3}$ para pasar, en un análisis de mayor profundidad, a describir cuál era el contenido de los comentarios de los usuarios a una noticia determinada sobre el 23-F. 
Para ello, además de verificar cuál era la información que sobre dicho hecho publicaban los medios tradicionales, se incluyó el portal de ranking editorial español Menéame.net ${ }^{4}$ con el objetivo de tratar de verificar cuál era la noticia más valorada del 23-F por los usuarios, y añadir así la visión recogida de una herramienta colaborativa propia de internet que, tal y como explican Orduña-Malea y Ontalba-Ruipérez (Orduña-Malea \& Ontalba-Ruipérez, 2008), permite a los usuarios registrados enviar noticias de periódicos digitales, de blogs o de otra fuentes de información disponibles en Internet, por tanto, no se publica la noticia sino el enlace a la fuente principal y una vez remitida, pasa a un listado de "pendientes" que, según los votos que obtenga, podrá pasar a la página principal donde alcanzará una mayor visibilidad y, por ende, un mayor número de visitas.

Las autoras de estas líneas detectaron que la mayoría de los medios de comunicación en su versión tradicional -especialmente notorio fue el caso de las cadenas de televisión: TVE y Antena 3- recurrían ese día (23 de febrero de 2011) al recuerdo de aquel hecho histórico a la par que esta rememoración servía de percha para hacer una comparación entre la España de la Transición y la actual y terminar, de este modo, destacando el progreso que en todos los ámbitos habría vivido el país durante las últimas tres décadas. ${ }^{5}$

En cualquier caso, la mayoría de los medios tradicionales transmitieron en sus informativos mensajes políticamente correctos y no desviados en ningún caso de la conocida versión oficial del 23-F que apenas ha evolucionado en estos últimos treinta años. $^{6}$

\footnotetext{
${ }^{4}$ Dentro de las herramientas de deliberación que han surgido por la proliferación de la web 2.0 destacan aquellos sitios web que han ido surgiendo a partir del británico Digg con gran popularidad entre los usuarios donde éstos envían relatos de noticias captadas en otros sitios en Internet para luego ser mostrados en una única página. Los usuarios votan y comentan estos relatos y, en función de su valoración, el relato ocupa un lugar de mayor o menor visibilidad dentro del sitio web; dando lugar así a lo que podría llamarse un portal de ranking editorial, como es el caso del portal menéame.com analizado aquí.

${ }^{5}$ Por ejemplo, Antena 3 arranció el informativo especial que emitió ese día resaltando el "cambio social" que había experimentado la sociedad española en los últimos 30 años, y por su parte, TVE dedicó la parte más destacada de su información sobre dicho aniversario a un reportaje breve en el personas de diferentes generaciones incidían en la evolución que España había vivido desde 1981 desde el punto de vista de la igualdad entre sexos, la economía, los derechos y libertades, etc.

${ }^{6} 6$ Por ejemplo, en el informativo de Antena 3 se insiste, dentro del programa especial que emitió ese día del año 2011 sobre el aniversario del 23F en que "se conoce toda la verdad" sobre los hechos acaecidos ese día e incluso llega a afirmar que no se llega a cuestionar en ningún momento que la
} 
Esta versión oficial y tradicional sitúa en un primer plano al monarca como "piloto del cambio" (Powell, 1991) -a quien considera una de los factores decisivos para el fracaso del golpe (Soto Carmona, 1998, p. 108)- y, en palabras de André-Bazzana "autor de la historia que condujo a España a la democracia" (André-Bazzana, 2006, p. 9); puesto que los golpistas no sólo fracasaron en la puesta en práctica de sus planes sino que además la recién nacida democracia española se acabó viendo reforzaba a la par que los apoyos hacia la persona de Juan Carlos I, hasta ese momento reducidos, crecieron y se afianzaron.

Así pues, este episodio de la Transición se saldó definitivamente con un efecto contrario al que se proponían sus ejecutores: "conjuró muchas amenazas que cernían sobre la nueva Constitución, contribuyó a afianzar la monarquía y salvó la democracia" (Carcedo, 2001, p. 384).

No obstante, el 23 de febrero de 2011 el periódico Publico, en un intento por desmarcarse de dicha versión oficial, divulgó una entrevista en la que se señalaba que "el 23-F acabó con una transición que podría haber llevado esta democracia muchísimo más lejos de donde está ahora". Se trata de una entrevista realizada al catedrático de Opinión Pública y Comunicación Política de la URJC Víctor Sampedro, quién ponía nuevamente sobre la mesa el debate la versión no oficial del 23-F.7

Esta noticia, reflejo de un posible surgimiento de un núcleo duro en relación a esa versión oficial de lo acaecido aquel 23-F, se convirtió el mismo día 23 de febrero de 2011 -y permaneció durante varios días- en la más popular del portal de ranking editorial español Menéame.net. ${ }^{8}$ Esta noticia recibió durante ese mismo día 23 de febrero un total de 44 comentarios de usuarios en la plataforma digital del diario Público y un total de 55 en el mismo espacio temporal en Menéame.net.

\subsection{Radiografía de la espiral del silencio en la web 2.0 el aniversario del 23-F}

Tomando como objeto de estudio el contenido de estos comentarios a la noticia en ambas plataformas -tanto en el diario Público como en Menéame.net- esta investigación persigue detectar hipótesis de trabajo que, teniendo como eje central la espiral de silencio, pueden despertar interrogantes que abran futuras investigaciones. Por tanto, este trabajo pretende configurarse como una primera aproximación para tratar de aportar, a los estudios de audiencias en Internet, algún fundamento a nuevas preguntas de investigación para los estudios de comunicación.

\footnotetext{
${ }^{7}$ En la referida entrevista, Sampedro afirma: “No se puede sostener al mismo tiempo que Tejero era un payaso sin ningún tipo de apoyo y que el monarca abortó un golpe de estado de consecuencias trágicas. Una cosa u otra.
} 
Es importante aclarar que las autoras de este trabajo que se presenta estiman que los 99 comentarios analizados no permiten establecer conclusiones finales sobre si, también en Internet y tal y como propugna la espiral del silencio, los usuarios tienen a difundir las ideas que se asemejen a la opinión mayoritaria, sino que el objetivo de esta investigación es realizar un primera aproximación al contenido de los mensajes realizados por los usuarios con el fin de tratar de establecer nuevas hipótesis de trabajo.

Para proceder al estudio de estos comentarios, se analiza el contenido y secuencia de todos los publicados por los lectores sobre esta noticia en base a la siguiente tipología planteada por León (2005):

a) Mensajes apoyatorios: se tratan de mensajes de apoyo a la noticia y a la fuente que o bien son laudatorios y de agradecimiento ${ }^{9}$ o bien amplían información en la línea de la noticia. ${ }^{10}$

b) Mensajes contrarios: figuran los contrarios a la noticia y a la fuente, que o bien su contenido es despreciativo ${ }^{11} \mathrm{o}$ argumentan una posición contraria a la manifiesta en la noticia. ${ }^{12}$

c) Mensajes neutros, aquellos indefinidos en su postura (ni a favor ni en contra), ${ }^{13}$ incomprensibles o cuyo mensaje que no está relacionado con el contenido de la noticia.

Atendiendo a las categorías anteriormente referidas, se puede considerar que los mensajes analizados estaban polarizados y que apenas se perciben diferencias cuantitativas significativas en el tipo de contenido de los comentarios. Así, aunque

9 Como ejemplo sirva el siguiente: "Estoy completamente de acuerdo, por lo menos una persona coherente y que dice la verdad, gracias por el artículo en nombre mío y de todos los demócratas de bien". Publicado en Público, 23 de febrero de 2011, 18:03. Disponible en: http://www.publico.es/espana/362746/victorsampedro-sin-el-golpe-estariamos-en-un-estado-federal [Última consulta: 8 de noviembre de 2011]

${ }^{10}$ Véase: "En este artículo Pujol explica algo parecido, que el 23-f significó un freno al proceso autonómico: $\quad$ http://www.elpuntavui.cat/noticia/article/3-politica/17-politica/374300-lesperit-de. Publicado en Menéame.net, 23 de febrero de 2011, 15:19. Disponible en: http://www.meneame.net/story/sin-golpe-estado-estariamos-estado-federal [Última consulta: 8 de noviembre de 2011]

11 Por ejemplo: "Las teorías son como los culos, todos tienen una. Y total, como siempre quedan muchas cosas en el aire, pues da para fantasear con 1001 teorías diferentes... Igual hay quien pueda argumentar que Elvis Presley planteaba hacerse cargo de España y tener como segunda dama a Marilyn Monroe, con ayuda de los ovnis" Publicado en Menéame.net, 23 de febrero de 2011, 15:03. Disponible en: http://www.meneame.net/story/sin-golpe-estado-estariamos-estado-federal [Última consulta: 8 de noviembre de 2011]

12 Véase: "Me parece un buen análisis el de D. Víctor Sampedro, aunque no estoy del todo de acuerdo con él en 
ligeramente predominan los mensajes contrarios, también son abundantes los mensajes neutros y significativos los apoyatorios [Gráfico 1].



Figura 1. Comentarios recibidos. Fuente: Elaboración propia

Las diferencias se aprecian observando el tipo de comentario por medio; puesto que los comentarios apoyatorios redominan en Público mientras que los neutros y contrarios son mayoritarios en Menéame.net Gráfico 2]. Es decir, los mensajes contarios a la noticia y fuente de la noticia se producen mayoritariamente cuando la noticia es comentada en la plataforma distinta a la raíz.

Se aprecia claramente que en Público la opinión mayoritaria coincide con el argumento manifestado por el entrevistado, incluso 4 de los 44 comentarios publicados en dicho website permanecen invisibles en la página principal de la noticia con la siguiente indicación: "Comentario oculto por la valoración de los lectores" como opción del medio -aunque también permite su lectura clicando en"Ver comentario" $^{\prime \prime}$, y todos estos son contrarios a la fuente y a la noticia. ${ }^{14}$ 


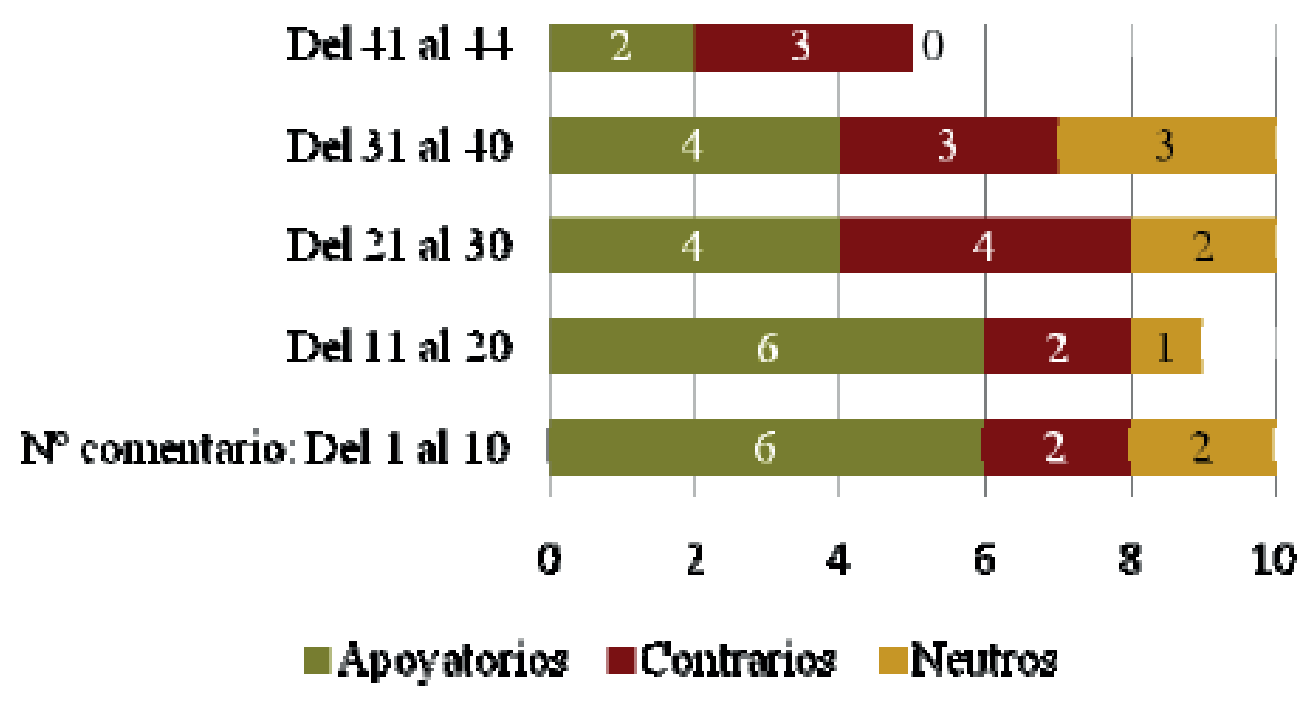

Figura 2. Tipo de comentarios recibidos por medio. Fuente: Elaboración propia

En cambio, los comentarios registrados en Menéame.net muestran claramente en su contenido -y especialmente acuciado según incrementa el número de ellos- que el debate se centra más en la discusión y pregunta-respuesta entre los propios internautas que en aportaciones al debate de fondo. Aunque, curiosamente, las alusiones y referencias directas entre los propios participantes es mayor en la plataforma de Público -8 de los 44 comentarios incluyen alusiones directas a otros participantes, generalmente con insultos y alusiones ofensivas, frente a sólo 2 de los 55 comentarios recogidos en Menéame.net que incluyen alusiones directas-.




Especialmente interesante es observar la tendencia del tipo de comentarios. La línea secuencial muestra que si bien mientras los primeros mensajes son apoyatorios en ambas plataformas -en mayor número, en Público-, a partir del mensaje número 19 en el diario [Gráfico 3] y del número 9 en Menéamenet [Gráfico 4] la tendencia se invierte y claramente pasan a predominar los comentarios contrarios.

Atendiendo a los primeros comentarios no apoyatorios de Público, los primeros contrarios no resultan especialmente polémicos ${ }^{15}$ pero a partir del comentario señalado anteriormente, la tendencia se invierte y comienzan a frecuentar los mensajes despectivos y con insultos. La misma tendencia se aprecia en Menéame.net: si bien los primeros comentarios contrarios resultan sutiles, ${ }^{16}$ también la tendencia se invierte con comentarios más extremistas y basados en ataques directos a la fuente. ${ }^{17}$

\footnotetext{
${ }^{15}$ Por ejemplo: "Sin el golpe de Estado estaríamos en un estado federal. Esto que plantea el señor Sampedro es una ucronía que al fin y al cabo no es más que una especulación sobre alternativas ficticias a los hechos realmente acaecidos. Como actividad recreativa puede tener cierto interés, pero su validez en el contexto del análisis histórico es nula". Publicado en Público, 23 de febrero de 2011, 19:01. Disponible en: http://www.publico.es/espana/362746/victor-sampedro-sin-el-golpe-estariamos-en-un-estadofederal [Última consulta: 8 de noviembre de 2011]

16 “Estaríamos igual de puteados, nos habrían hecho los recortes salariales igual y la jubilación a los 67. Sólo que entonces nos habrían dicho que hay que hacer un esfuerzo por el futuro..." Publicado en Menóame net. 73 de fehrern de 011 . 16.4ח Disnonihle en. httn / / wrurw meneame net/storv/sin-onlne-
} 


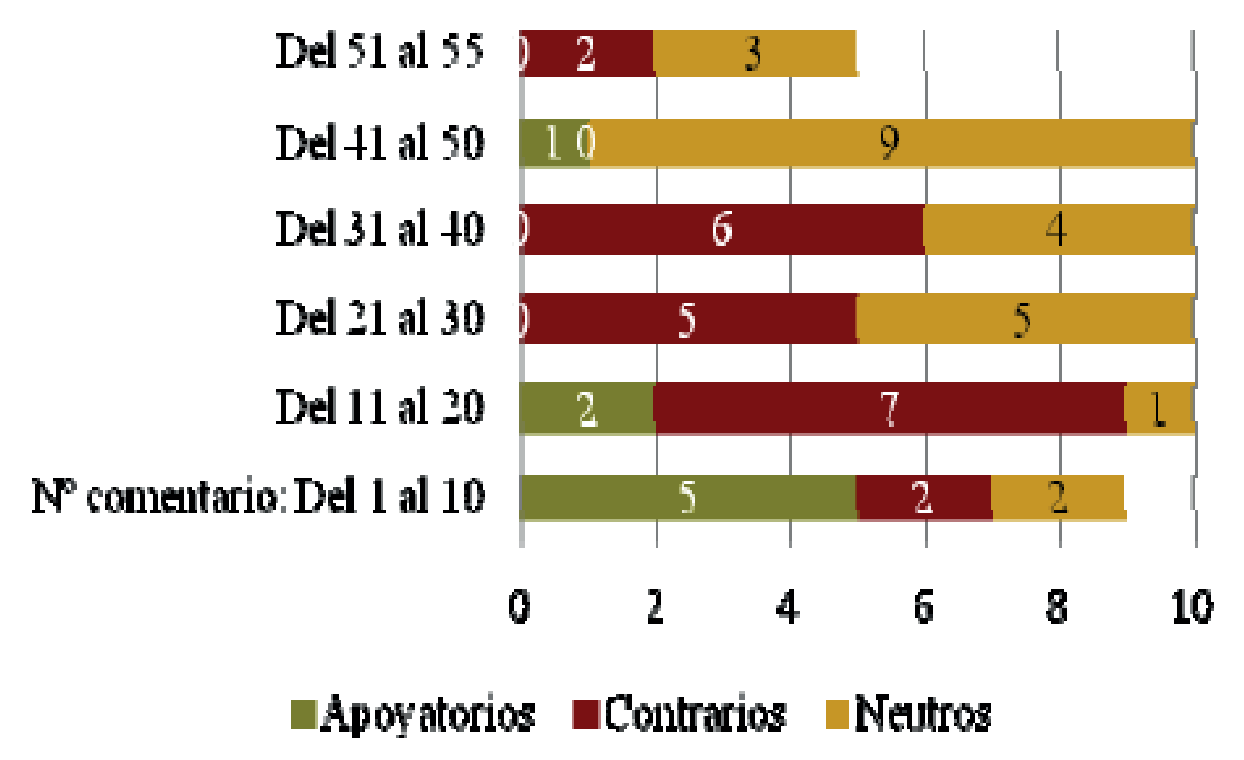

Figura 4. Evolución del tipo de comentarios recibidos en Menéame.net.

Por tanto, los datos sí parecen mostrar que cuando afloran los comentarios más radicales y opiniones basadas más en el insulto que en la discusión -al ser considerados por los usuarios como la vanguardia representante de una opinión respaldada por una posible mayoría- estos provoquen que la corriente de opinión se invierta; con lo cual estos mensajes ejercen de punto de inflexión para que los otros usuarios se excluyan del proceso de discusión y, como consecuencia, caigan en la espiral del silencio.

\section{CONCLUSIONES}

A partir del análisis que se ha ido desarrollando en estas páginas, y teniendo siempre en consideración las limitaciones de esta investigación que han sido señaladas previamente, podemos formular las conclusiones del trabajo; las cuales pretenden ofrecer al lector nuevos interrogantes que despierten el interés por abordar novedosos estudios en el ámbito de la comunicación: 
Como primer punto de estas conclusiones podemos afirmar que efectivamente los comentarios de los lectores publicados en ambas plataformas analizadas presentan una línea de pensamiento semejante, coincidiendo con el mensaje y fuente de la noticia en un primer término pero, a medida que discurre el debate y se introduce un actor desviado que rompe esa consonancia, comienzan a publicarse comentarios que le respaldan y, por tanto, aparecen opiniones contrarias al mensaje y a la fuente de la noticia. ${ }^{18}$

En segundo lugar, tal y como ya han formulado otros autores, los insultos promueven, en este estudio, en el discurso político más respuestas contrarias entre los propios participantes de la comunidad que estimulan el contra-ataque, de tal forma que:

El maltrato verbal mediático-político está teniendo en la impunidad, como otrora ocurriera con el de género, el mobbing o el bullying, y no es menos destructivo. Aunque apenas hay estudios al respecto bajo esta terminología, aportaciones como el modelo de la espiral del silencio apuntan a que es un método eficaz para neutralizar incluso a mayorías sociales de opinión por parte de minorías" (León, 2005, P173).

Por ello, formulamos una segunda hipótesis de trabajo futura: El punto de inflexión entre los comentarios a favor de la fuente y del mensaje de la noticia se produce cuando los usuarios

En tercer lugar, los comentarios a las noticias en los medios digitales son, a día de hoy, todavía espacios más de desahogo o expresión del ciudadano que verdaderos espacios de participación ciudadana, donde no existen catalizadores y el fin de dicho debate no está explícito.

Actualmente, los espacios destinados a los comentarios de las noticias que pueden enviar los usuarios son considerados como baúles abiertos para que ciudadanos, escudados generalmente en el anonimato, 19 expresen sus opiniones. Sin embargo, es importante destacar que entre todas las opiniones que llegan a verterse, aquellas que se basan en posiciones más radicalizadas y extremistas van a ser percibidas por la audiencia como si fuesen un mensaje lanzado por la vanguardia de la sociedad.

\footnotetext{
${ }^{18}$ Las limitaciones de esta investigación no permiten, en esta publicación, conocer si estas opiniones provienen de aquellos ciudadanos que por miedo al ostracismo había optado por el silencio y sólo se manifiestan cuando su opinión resulta manifiesta por otro actor. formulan insultos y ello promueve que
} 
Es decir, esas voces son escuchadas por el resto de los usuarios como si se tratase de mensajes provenientes de un portavoz de la opinión generalizada. Por lo tanto, esos comentarios más radicales terminan dominando ese espacio de opinión en detrimento de otras posturas más consensuadas, basadas en la discusión y no en el insulto, que podrían beneficiar la participación ciudadana a través de la empatía y no en ausencia de claves sociales.

Teniendo en cuenta esta percepción de los usuarios, es preciso que las investigaciones sobre comunicación ahonden aún más en el efecto que puede producir el anonimato dentro del proceso de configuración de la opinión pública a través de las comunidades virtuales de los medios en Internet, y, con ello, poder analizar con mayor precisión el fenómeno de la exclusión y aislamiento que se genera en torno a los individuos dentro de espacios de debate como son los habituales comentarios a las noticias.

\section{REFERENCIAS}

André-Bazzana, B. (2006). Mitos y mentiras de la Transición. Madrid: El Viejo Topo.

Carcedo, D. (2001). 23F. Los cabos sueltos. Madrid: Temas de Hoy.

Castells, M. (2010). Comunicación y poder. Madrid: Alianza Editorial.

Monzón, C. (2006). Opinión pública, comunicación y política. Madrid: Tecnos.

Noelle-Neumann, E. (2003). La espiral del silencio. Opinión pública: nuestra piel social. Barcelona: Paidós Comunicación.

Powell, Ch. T. (1991). El piloto del cambio. El Rey, la monarquía y la transición a la democracia. Barcelona: Planeta.

Soto Carmona, A. (1998). La transición a la democracia. España 1975-1982. Madrid: Alianza editorial.

Noelle-Neumann, E. (1978). El doble clima de opinión, la influencia de la televisión en una campaña electoral. Reis, 41.

Noelle-Neumann, E. (1979). Public Opinion and the Classical Tradition: A Revaluation. Public Opinion Quarterly, 43(2): 143-156. 
Herrero, J. et al. (2004). Participación social en contextos virtuales. Psicothema, 16(3): 456-460. Recuperado el 9 de noviembre de 2011, de http://www.psicothema.com/pdf/3018.pdf.

León, J. L. (2005). La oposición a mensaje y fuente en los nuevos contextos persuasivos de Internet. Comunicación, 3. Recuperado el 9 de noviembre de 2011, de http://www.revistacomunicacion.org/pdf/n3/articulos/la_oposicion_a_mensaje_y _fuente_en_los_nuevos_contextos_persuasivos_de_internet.pdf.

López García, G. (2011). La relación de los periodistas con los lectores. Gestión de la participación del público, sistemas de moderación y modelos de espacio público. En Libertades de expresión e información en Internet y las redes sociales: ejercicio, amenazas y garantías. Recuperado el 9 de noviembre de 2011, de http://www.uv.es/cotino/elibertades2010.pdf.

Rojas, F. \& Hopke, J. (2010). Socializados para la autocensura: Comunicación autoritaria y opinión pública. Revista Latinoamericana de Opinión Pública, 1(0): 165-173. Recuperado el 9 de noviembre de 2011, de http://www.waporlatinoamerica.org/descargas/revista/n0/rojas_socializados.pdf.

\section{Virginia Martín Jiménez}

Doctora por la Universidad de Valladolid (con título de doctorado europeo), licenciada en Periodismo, licenciada en Historia y diplomada en Sociología, Virginia Martín Jiménez es profesora de Periodismo en la Facultad de Filosofía y Letras de la Universidad de Valladolid. Sus líneas de investigación se enmarcan en el ámbito de la comunicación política, los estudios sobre la Transición democrática, la historia de la comunicación social y la conformación de la opinión pública a través de los medios de comunicación. Ha sido investigadora invitada en centros internacionales de reconocido prestigio como la Universidad Carolina de Praga, el Centro de Estudios de la Población, Economía y Sociedad de la Universidad de Oporto o la Universidad Nova de Lisboa. Ha publicado en editoriales como Routledge y Fragua, pasando por otras editoriales de tipo institucional o académico. Sus investigaciones han sido recogidas por revistas indexadas de impacto internacional como la británica Journal of Spanish Cultural Studies o la especializada Historia Actual online. A lo largo de su carrera como investigadora ha disfrutado de prestigiosas becas de investigación tales como la Beca de Formación de Profesorado Universitario del Ministerio de Educación.

\section{Eva Campos Domínguez}

